\title{
A Case Report of Ischemic Stroke in a Patient with Metastatic Gastric Cancer Secondary to Treatment with the Vascular Endothelial Growth Factor Receptor-2 Inhibitor Ramucirumab
}

\author{
Michael E. Christiansen ${ }^{\mathrm{a}}$ Timothy Ingall $^{\mathrm{b}}$ Edward C. Lew \\ Ramesh K. Ramanathan $^{d}$ Harshita R. Paripati $^{d}$ \\ ${ }^{a}$ Mayo Clinic School of Graduate Medical Education, Mayo Clinic Arizona, \\ Phoenix, Ariz., USA; ${ }^{b}$ Department of Neurology, Mayo Clinic Arizona, Phoenix, Ariz., USA; \\ 'Division of Hematology/Oncology, Department of Medicine, Mayo Clinic Arizona, \\ Phoenix, Ariz., USA; ${ }^{d}$ Division of Hematology/Oncology, Department of Medicine, Mayo \\ Clinic Arizona, Phoenix, Ariz., USA
}

\section{Keywords}

Angiogenesis inhibitors · Gastric cancer $\cdot$ Ischemic stroke $\cdot$ Adverse effects

\section{Abstract}

Ramucirumab is an antiangiogenesis agent targeting the vascular endothelial growth factor receptor-2 (VEGFR-2), approved to treat advanced gastric and colon cancer. In clinical trials, it was shown to cause a small increase in arterial thromboembolism compared to placebo, including cerebral and myocardial ischemia, which was not statistically significant. Detailed case reports are lacking and we here present one of the first case reports of stroke secondary to ramucirumab-induced in situ thrombosis. 


\section{Case Reports in Oncology}

\section{Introduction}

Ramucirumab is a recombinant monoclonal antibody that binds to vascular endothelial growth factor receptor-2 (VEGFR-2), and is approved for use in advanced gastric, lung, and colon cancer. Pivotal studies in gastric cancer have shown that ramucirumab as a single agent or in combination with paclitaxel prolonged overall survival. In the RAINBOW trial, patients with advanced gastric cancer (disease progression after 4 months of first-line therapy with platinum plus fluoropyrimidine) who received ramucirumab plus paclitaxel ( $\mathrm{n}=$ 330) demonstrated significantly prolonged median survival compared to the paclitaxel plus placebo group ( $n=335 ; 9.6$ vs. 7.4 months, $p=0.017$ ) [1]. In the REGARD trial, ramucirumab single-agent therapy $(\mathrm{n}=238)$ also significantly extended median survival compared to placebo $(\mathrm{n}=117)$ in patients with advanced gastric cancer (5.2 vs. 3.8 months, $\mathrm{p}=0.047$ ) [2]. Arterial thromboembolism (TE) occurred slightly more frequently in the ramucirumab arm, but the difference was not statistically significant $(2 \%$ any grade and $1 \%$ grade 3 or higher vs. $0 \%$ placebo, $p=0.55$ ) [2]. Though reported as an adverse event in publications of the clinical trials and the product brochure [3], to the best of our knowledge, this is the first case report detailing the clinical manifestations and workup of a patient suspected of having a cerebral TE event associated with ramucirumab.

\section{Case Report}

An 82-year-old male patient with a diagnosis of metastatic gastric cancer presented to the emergency department after an episode of confusion and fall. Until 2 weeks prior to this event, the patient was functionally independent with a performance status of ECOG 1, but recently displayed increasing confusion, unsteady balance, numerous falls, and sometimes difficulty with speech. There was no significant tobacco, alcohol, or recreational drug use history. His past medical history was significant for peripheral neuropathy affecting his feet, hypertension, and hypothyroidism. He was diagnosed with gastric cancer 11 months prior by stomach wall biopsy demonstrating poorly differentiated adenocarcinoma with signet ring cells. Staging CT scans showed metastases to the omentum, retroperitoneum, diaphragmatic lymph nodes, and malignant ascites. He initially began standard chemotherapy with carboplatin and paclitaxel, with a partial response which lasted for several months. Six months later, he was switched to capecitabine due to disease progression, which was poorly tolerated and discontinued after 1 cycle. Subsequently he received ramucirumab singleagent therapy at the dose of $8 \mathrm{mg} / \mathrm{kg}$ given intravenously. He received four doses, each spaced 2 weeks apart, with the last dose 2 weeks prior to presentation.

His physical exam was significant for orientation to person only and ataxia on the fingernose-finger test. Initial laboratory results were within normal limits except for creatinine 1.6 $\mathrm{mg} / \mathrm{dl}, \mathrm{BUN} 30 \mathrm{mg} / \mathrm{dl}$, and TSH $14.24 \mathrm{mIU} / \mathrm{l}$ with normal T4 $1.2 \mathrm{ng} / \mathrm{dl}$. Cardiac telemetry did not show any evidence of arrhythmia. CT of the head did not show any acute changes to explain the patient's symptoms. Carotid Doppler ultrasound was negative for significant stenosis. Transthoracic and transesophageal echocardiogram was negative for thrombus or severe atrial dilation; it did note a patent foramen ovale with a very small amount of right-toleft shunting. MRI with gadolinium of the brain revealed multifocal mildly increased diffusion signals with a corresponding increased T2 and FLAIR signal, likely subacute, involving the bilateral cerebellar hemispheres, bilateral occipital lobes, and bilateral parietal cortex (fig. 1), confirming multifocal ischemic stroke, while in the hospital the patient underwent 


\section{Case Reports in Oncology}

hysical and occupational therapy and did achieve improvement in his symptoms. However, due to his poor prognosis and overall decline in functional status it was decided by the patient and his family to be discharged home with hospice.

\section{Discussion}

Angiogenesis inhibitors are used in certain types of cancer to block neovascularization for rapid tumor growth. In a systematic review of adverse outcomes associated with VEGF inhibitors, Faruque et al. [4] report an increase in thrombotic events which is not statistically significant: $227 / 1,897$ experienced any thrombosis versus 144/1,850 controls (RR 1.53, 95\% CI 1.07-2.20, $\mathrm{p}=0.07$ ), and 89/3,503 experienced arterial thrombosis compared to $42 / 2,741$ controls (RR 1.80, 95\% CI 1.24-2.59, $\mathrm{p}=0.67$ ). Two studies in the review testing bevacizumab plus axitinib coadministered with gemcitabine in pancreatic cancer patients reported combined rate of fatal and nonfatal stroke of 7/582 with VEGF inhibitor therapy and 6/571 with control therapy (RR 1.12, 95\% CI 0.38-3.30, p = 0.94) [4-6]. Despite this lack of statistical significance, there are case reports of stroke thought to be due to bevacizumab [7]. It is difficult to determine the attributable risk of stroke due to the use of VEGF inhibitors in these cases as they are rare.

In the RAINBOW trial, ramucirumab plus paclitaxel did not carry any increase in arterial TE risk compared to placebo plus paclitaxel [1]. However, in the REGARD trial with ramucirumab monotherapy, 4/236 patients randomized to ramucirumab experienced an arterial TE event, compared to $0 / 115$ on placebo [2]. This category of adverse event broadly included angina pectoris, cardiac arrest, cerebral ischemia, cerebrovascular accident, myocardial infarction, or myocardial ischemia. Ischemic stroke is divided into five categories based on likely etiology: large artery atherosclerosis, cardioembolism, small vessel occlusion, stroke of other determined etiology, and stroke of undetermined etiology [8]. Although the spatial distribution and the age of these strokes are consistent with an embolic pattern, no source of embolus could be identified. It appeared likely that the strokes were most likely due to in situ thrombosis, related to the prothrombotic side effects of ramucirumab. In this case, the patient's risk could have been further increased by his hypercoagulable state due to metastatic disease, advanced age, and history of hypertension. Physicians must be appropriately educated regarding the potential morbidity and mortality associated with increased stroke risk, especially in patient groups with other risk factors for arterial TE events.

\section{Statement of Ethics}

Informed consent was obtained for publication of this case.

\section{Disclosure Statement}

The authors have no conflicts of interest to disclose. 


\section{Case Reports in Oncology}

\begin{tabular}{l|l}
\hline Case Rep Oncol 2016;9:317-320 \\
\hline $10.1159 / 000446695$ & $\begin{array}{l}\text { (c) 2016 The Author(s). Published by S. Karger AG, Basel } \\
\text { www.karger.com/cro }\end{array}$ \\
\hline
\end{tabular}

Christiansen et al: A Case Report of Ischemic Stroke in a Patient with Metastatic Gastric Cancer Secondary to Treatment with the VEGFR-2 Inhibitor Ramucirumab

\section{References}

1 Wilke H, Muro K, Van Cutsem E, et al: Ramucirumab plus paclitaxel versus placebo plus paclitaxel in patients with previously treated advanced gastric or gastro-oesophageal junction adenocarcinoma (RAINBOW): a double-blind, randomized phase 3 trial. Lancet Oncol 2014;15:1224-1235.

-2 Fuchs C, Tomasek J, Yong C, et al: Ramucirumab monotherapy for previously treated advanced gastric or gastro-oesophageal junction adenocarcinoma (REGARD): an international, randomized, multicenter, placebo-controlled, phase 3 trial. Lancet 2014;383:31.

3 Cyramza, ramucirumab injectable solution, online product brochure. http://www.cyramzahcp.com/gastric/safety/rainbow-adverse-reactions.html. Accessed Janurary 28, 2016.

4 Faruque L, Lin M, Battistella M, Wiebe N, Reiman T, Hemmelgarn B, Thomas C, Tonelli M: Systematic Review of the Risk of Adverse Outcomes Associated with Vascular Endothelial Growth Factor Inhibitors for the Treatment of Cancer. PLOS One 2014;9:e101145.

-5 Kindler HL, Niedzwiecki D, Hollis D, Sutherland S, Schrag D, Hurwitz H, Innocenti F, Mulcahy MF, O’Reilly E, Wozniak TF, Picus J, Bhargava P, Mayer RJ, Schilsky RL, Goldberg RM: Gemcitabine plus bevacizumab compared with gemcitabine plus placebo in patients with advanced pancreatic cancer: phase III trial of the Cancer and Leukemia Group B (CALGB 80303). J Clin Oncol 2010;28:3617-3622.

-6 Kindler HL, Ioka T, Richel DJ, Bennouna J, Letourneau R, Okusaka, T, Funakoshi A, Furuse J, Park YS, Ohkawa S, Springett GM, Wasan HS, Trask PC, Bycott P, Ricart AD, Kim S, Van Cutsem E: Axitinib plus gemcitabine versus placebo plus gemcitabine in patients with advanced pancreatic adenocarcinoma: a double-blind randomized phase 3 study. Lancet Oncol 2011;12:256-262.

7 Domenico G, Vincenza T, Emiddio B, Dino T, Franco M, Bruno D: A Fatal Brain Stroke in Patient with Advanced Breast Cancer Treated with Bevacizumab: A Case Report. World J Oncol 2010;1:252-254

$\checkmark 8$ Adams HP Jr, Bendixen BH, Kappelle LJ, et al: Classification of subtype of acute ischemic stroke. Definitions for use in a multicenter clinical trial. TOAST. Trial of Org 10172 in Acute Stroke Treatment. Stroke 1993;24:35.
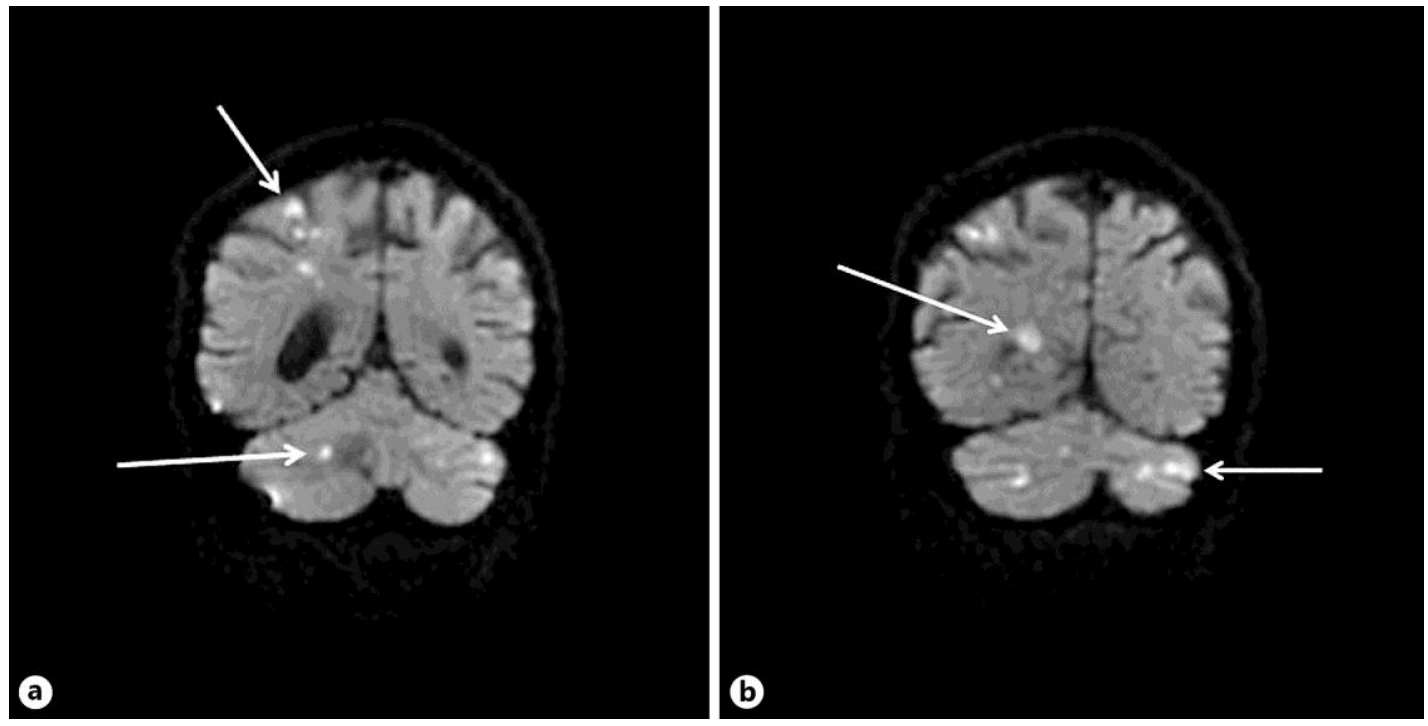

Fig. 1. Axial MR images of the brain showing diffusion restriction (a) and FLAIR T2 hyperintensity (b) corresponding to areas of ischemic infarction. Multifocality and subacute appearance are consistent with in situ thrombosis versus TE. 